\title{
Sensor Data Fusion Based on a New Conflict Measure
}

\author{
Wen Jiang, Boya Wei, Xiyun Qin, Jun Zhan, and Yongchuan Tang \\ School of Electronics and Information, Northwestern Polytechnical University, Xian, Shaanxi 710072, China \\ Correspondence should be addressed to Wen Jiang; jiangwen@nwpu.edu.cn
}

Received 24 January 2016; Revised 10 April 2016; Accepted 17 April 2016

Academic Editor: Jianbing Ma

Copyright (C) 2016 Wen Jiang et al. This is an open access article distributed under the Creative Commons Attribution License, which permits unrestricted use, distribution, and reproduction in any medium, provided the original work is properly cited.

Dempster-Shafer (D-S) evidence theory has been widely used in various fields. However, how to measure the degree of conflict (similarity) between the bodies of evidence is an open issue. In this paper, in order to solve this problem, firstly we propose a modified cosine similarity to measure the similarity between vectors. Then a new similarity measure of basic probability assignment (BPAs) is proposed based on the modified cosine similarity. The new similarity measure can achieve the reasonable measure of the similarity of BPAs and then efficiently measure the degree of conflict among bodies of evidence. Numerical examples are used to illustrate the effectiveness of the proposed method. Finally, a weighted average method based on the new BPAs similarity is proposed, and an example is used to show the validity of the proposed method.

\section{Introduction}

Uncertainty information modeling and processing are still an open issue. To address this issue, many mathematical tools are presented like fuzzy sets theory [1-5], evidence theory [6-9], rough sets theory [10-12], $Z$ numbers [13-16], $D$ numbers theory $[17,18]$, and so on [19]. Decision making and optimization under uncertain environment are heavily studied [20-23]. Due to the efficiency modeling and fusion of information, evidence theory is widely used [24,25]. And sometimes, the methods with mixed intelligent algorithms are used for decision making or related problems $[20,25,26]$.

Dempster-Shafer (D-S) evidence theory [6,7] provides a natural and powerful way for the expression and fusion of uncertain information, so it has been widely used in various fields of information fusion $[9,27,28]$, target recognition [29, 30], decision making [31], image processing [32], uncertain reasoning [33], and risk analysis [25, 26, 34, 35]. However, the counterintuitive results may be obtained by conventional combination rule when collected bodies of evidence highly conflict with each other [36]. For example, in the real battle field, because of hard natural factors and human interference, the information obtained by sensor tends to have high uncertainty and high conflict with other pieces of information. If this problem cannot be solved effectively, it will greatly limit the application of D-S evidence theory. Many scholars have conducted in-depth research and put forward a number of enhancements to the evidence combination algorithm $[8,24$, 37-39]. In general, the existing methods of the conflict information can be divided into two categories. One method is to modify Dempster's combination rule. When $k=1$, the rule is not applicable. It solves the problem that how to redistribute and manage the conflict by modifying the rule. Another method is to modify the data model. The conflict evidence is preprocessed first and then is combined by combination rules.

Obviously, it is a critical issue to determine the degree of conflict between bodies of evidence. Researchers usually use conflict coefficient $k$ to indicate the degree of conflict. Whereas $k$ only reflects the noninclusive between bodies of evidence. For example, if two bodies of evidence are in certain conflict, the value of $k$ may be 0 . Obviously, the result is incorrect. The classical conflict coefficient $k$ is not a pretty good conflict measure. In recent years, many works on this issue have emerged [40, 41]. In order to avoid a wrong claim made by using only $k$, in [41], pignistic distance $\operatorname{difBet} P$ is introduced by Liu and two-tuple variable $\langle k$, $\operatorname{difBetP}\rangle$ is proposed to measure the degree of conflict among BPAs. But Liu did not propose a formula to unify the two factors, since we often need a measured value of conflict when we process the conflict of evidence. Moreover, Jousselme distance $d_{j}$ [40] is also commonly used to measure the conflict of evidence, which reflects the difference of BPAs. Nevertheless it captures 
only one aspect of the dissimilarity among BPAs mainly associated with a distance metric. In [42] Deng et al. proposed a new relative coefficient to characterize the conflict between bodies of evidence. Although this coefficient solves some problems of $k$, there still exist some deficiencies. For example, it lacks physical meaning during the definition of relative coefficient and it is not equal to 0 when two BPAs are totally contradictory with each other. In order to indicate the conflict between bodies of evidence precisely, a new method to measure the degree of conflict is proposed in this paper.

In previous works, distance and angle are often considered in the similarity measurement between vectors [43]. However, both the angle-based similarity measurement and the distance-based similarity measurement cannot effectively indicate the similarity among vectors. If the two factors and other factors can be used jointly, better performance can be expected. According to such an idea, we proposed a modified cosine similarity based on distance, cosine angle, and ratio of vector modes to measure the similarity between vectors.

Furthermore, a new similarity of BPAs is proposed based on the modified cosine similarity, which is used to measure the degree of conflict. If two bodies of evidence are consistent with each other, we think that the similarity between them is high; it means that the degree of conflict among them is low. When the proposed similarity is close to 1 , the value indicates that there is little contradiction between bodies of evidence. When the proposed similarity is close to 0 , this value indicates that the two bodies of evidence are in high conflict.

This paper is organized as follows. Section 2 describes some basic concepts. Section 3 details the problem of existing conflict measurements between BPAs. In Section 4, we will investigate the new similarity of BPAs. Section 5 presents some examples and analysis is presented. In Section 6, an example is shown to illustrate the effectiveness of our method. Finally, some conclusions are summarized in Section 7.

\section{Preliminaries}

\subsection{The Basic Concept of D-S Evidence Theory [6, 7]}

Definition 1. Let $\Theta$ be a nonempty finite set which is called the frame of discernment; its power set containing all the possible subsets of $\Theta$ is denoted by $2^{\Theta}$, where the elements are mutually exclusive and exhaustive. Define the function $m: 2^{\Theta} \rightarrow[0,1]$ as the basic probability assignment (BPA) (also called a belief structure or a basic belief assignment), which satisfies

$$
\begin{aligned}
\sum_{A \subseteq \Theta} m(A) & =1 \\
m(\phi) & =0,
\end{aligned}
$$

where $m(A)$ is defined as the BPA of $A$, representing the strength of all the incomplete information sets for $A$.

If $m(A)>0$, then $A$ is called the focal element. The degree of one's belief to a given proposition is represented by a two-level probabilistic portrayal of the information set: the belief level and the plausibility level. They are defined as follows:

$$
\begin{aligned}
\operatorname{Bel}(A) & =\sum_{B \subseteq A} m(B), \quad \forall A \subseteq \theta \\
\operatorname{Pl}(A) & =1-\operatorname{Bel}(\bar{A})=\sum_{B \cap A \neq \phi} m(B), \quad \forall A \subseteq \theta,
\end{aligned}
$$

where $\operatorname{Bel}(A)$ is the sum of $m(B)$ for all subsets $B$ contained in $A$, representing all the bodies of evidence that support the given proposition $A . \operatorname{Pl}(A)$ which is the sum of $m(B)$ for all subsets $B$ has a nonnull intersection of $A$ and represents all the bodies of evidence that do not rule out the given proposition $A$. Absolutely, $\operatorname{Pl}(A)>\operatorname{Bel}(A)$. The belief interval $[\operatorname{Bel}(A), \operatorname{Pl}(A)]$ represents the uncertainty of $A$. When $\operatorname{Pl}(A)=\operatorname{Bel}(A)$, this means absolute confirmation to $A$.

Definition 2. Let $m_{1}$ and $m_{2}$ be two BPAs on the same frame of discernment $\Theta$. Dempster's combination rule is expressed as follows:

$$
m(A)= \begin{cases}\frac{\sum_{B \cap C=A} m_{1}(B) m_{2}(C)}{1-k} & A \neq \phi \\ 0 & A=\phi\end{cases}
$$

with

$$
k=\sum_{B \cap C=\phi} m_{1}(B) m_{2}(C)
$$

where $k$ is a normalization constant, which is called conflict coefficient of two BPAs.

According to the above formula, we can obtain that $k=0$ corresponds to the absence of conflict between $m_{1}$ and $m_{2}$, whereas $k=1$ implies complete contradiction between $m_{1}$ and $m_{2}$. The above rule is meaningful only when $k \neq 1$; otherwise the rule cannot be applied.

\subsection{Liu's Method [41]}

Definition 3. Let $m$ be a BPA on the frame of discernment $\Theta$. Its associated pignistic probability function $\operatorname{Bet}_{m}: \Theta \rightarrow$ $[0,1]$ is defined as follows:

$$
\operatorname{Bet}_{m}(w)=\sum_{A \in 2^{\Theta}, w \in A} \frac{1}{|A|} \frac{m(A)}{1-m(\phi)}, \quad m(\phi) \neq 1,
$$

where $|A|$ is the cardinality of proposition $A$.

Definition 4. Let $m_{1}$ and $m_{2}$ be two BPAs on frame $\Theta$ and let $\operatorname{Bet} P_{m_{1}}$ and $\operatorname{Bet} P_{m_{2}}$ be the results of two pignistic transformations from them, respectively. Then $\operatorname{difBet} P_{m_{2}}^{m_{1}}$ is defined as follows:

$$
\operatorname{difBet} P_{m_{2}}^{m_{1}}=\max _{A \subseteq \Theta}\left(\left|\operatorname{Bet} P_{m_{1}}(A)-\operatorname{Bet} P_{m_{2}}(A)\right|\right)
$$

which is called the distance between betting commitments of the two BPAs. 
Value $\left(\left|\operatorname{Bet} P_{m_{1}}(A)-\operatorname{Bet} P_{m_{2}}(A)\right|\right)$ is the difference between betting commitments to $A$ from the two sources. The distance of betting commitments is the maximum extent of the differences between betting commitments to all the subsets. $\operatorname{difBet} P_{m_{2}}^{m_{1}}$ is simplified as $\operatorname{difBet} P$.

Definition 5. Let $m_{1}$ and $m_{2}$ be two BPAs on frame $\Theta$ and let $c f\left(m_{1}, m_{2}\right)=\langle k, \operatorname{dif} \operatorname{Bet} P\rangle$ be a two-dimensional measurement. $m_{1}$ and $m_{2}$ are defined as in conflict, if both $\operatorname{difBet} P>\varepsilon$ and $k>\varepsilon$ hold, where $k$ is conflict coefficient and $\operatorname{dif} \operatorname{Bet} P$ is the distance between betting commitments in Definition 4 and where $\varepsilon \in[0,1]$ is the threshold of conflict tolerance.

\subsection{Correlation Coefficient [45]}

Definition 6. Let the distribution of random variables $X, Y$ be

$$
\begin{aligned}
& X:\left\{\begin{array}{l}
a_{1}, \ldots, a_{k} \\
p_{1}, \ldots, p_{k}
\end{array}\right\} \\
& Y:\left\{\begin{array}{l}
b_{1}, \ldots, b_{k} \\
q_{1}, \ldots, q_{k}
\end{array}\right\} .
\end{aligned}
$$

Then the partial entropy of $X$ on $Y$ is defined as follows:

$$
H_{Y}(X)=-\sum_{i=1}^{k} q_{i} \log p_{i} .
$$

The entropy of random variables is defined as follows:

$$
H(X)=-\sum_{i=1}^{k} p_{i} \log p_{i}
$$

Definition 7. The relative entropy of random variables $X, Y$ is defined as the sum of their partial entropy:

$$
H(X ; Y)=H_{Y}(X)+H_{X}(Y) .
$$

Definition 8. The partial correlation coefficient and correlation coefficient of random variables $X, Y$ are defined as follows:

$$
\begin{aligned}
\gamma_{Y}(X) & =\frac{H(Y)}{H_{Y}(X)} \\
\gamma_{X}(Y) & =\frac{H(X)}{H_{X}(Y)} \\
\gamma(X ; Y) & =\frac{H(X \otimes Y)}{H(X ; Y)}=\frac{H(X)+H(Y)}{H_{X}(Y)+H_{Y}(X)} .
\end{aligned}
$$

In [45], the authors have proved that the correlation coefficient has the following property:

$$
0 \leq \gamma_{Y}(X), \gamma_{X}(Y), \gamma(X ; Y) \leq 1
$$

If and only if the distribution of $X, Y$ is the same, then

$$
\gamma_{Y}(X)=\gamma_{X}(Y)=\gamma(X ; Y)=1 .
$$

This property indicates that the correlation coefficient $\gamma(X ; Y)$ is the characteristic metrics of identity and consistency of the distribution of random variables $X$ and $Y$.

In order to measure the degree of conflict between bodies of evidence, Deng et al. in 2011 [42] proposed a similar partial entropy, and it is defined as follows:

$$
H_{Y}(X)=\sum_{i=1}^{k} q_{i} * e^{-5 p_{i}}
$$

According to checking, the coefficient $e^{-5 p_{i}}$ in (14) is relatively close to log.

\subsection{Jousselme Distance [40]}

Definition 9. Let $m_{1}$ and $m_{2}$ be two BPAs on the same frame of discernment $\Theta . A_{i}$ and $B_{j}$ are focal elements of $m_{1}$ and $m_{2}$, respectively. The Jousselme distance, denoted by $d_{j}\left(m_{1}, m_{2}\right)$, is defined as follows:

$$
d_{j}\left(m_{1}, m_{2}\right)=\sqrt{0.5 *\left(\left\|m_{1}\right\|^{2}+\left\|m_{2}\right\|^{2}-2\left\langle m_{1}, m_{2}\right\rangle\right)},
$$

where $\left\|m_{1}\right\|^{2}=\left\langle m_{1}, m_{1}\right\rangle ;\left\|m_{2}\right\|^{2}=\left\langle m_{2}, m_{2}\right\rangle ;\left\langle m_{1}, m_{2}\right\rangle$ represents the scalar product of two vectors. It is defined as follows:

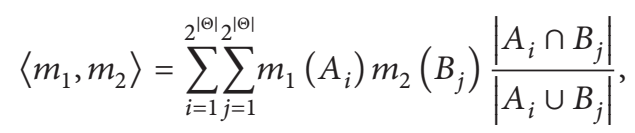

where $A_{i}$ and $B_{j}$ are the elements of framework $\Theta,\left|A_{i} \cap B_{j}\right|$ is the cardinality of common objects between elements $A_{i}$ and $B_{j}$, and $\left|A_{i} \cup B_{j}\right|$ is the number of subsets of union of $A_{i}$ and $B_{j}$.

\subsection{New Conflict Coefficient [46]}

Definition 10. The new conflict coefficient $k^{d}$ of two bodies of evidence is defined as follows:

$$
k^{d}=\frac{1}{2} \times\left(k+d_{j}\right),
$$

where $k$ is classical conflict coefficient and $d_{j}$ is the Jousselme distance. The larger the $k^{d}$ is, the larger the degree of conflict will be.

When both measurements are 0 , it is safe to say that there is no contradiction between $m_{1}$ and $m_{2}$. Only when both $k$ and $d_{\mathrm{BPA}}$ have a relatively high value, this pair of values indicates a strong conflict between $m_{1}$ and $m_{2}$. When both measurements have a relatively low value, this indicates that the two BPAs have little contradiction.

2.6. Cosine Similarity. There are two vectors $A=\left[a_{1}, a_{2}, \ldots\right.$, $\left.a_{n}\right], a_{i} \geq 0, i=0, \ldots, n$, and $B=\left[b_{1}, b_{2}, \ldots, b_{n}\right], b_{i} \geq 0$, $i=0, \ldots, n$. Due to the complexity in fault diagnosis $[47,48]$, evidence theory is used to handle sensor fusion to obtain the reasonable result [24]. It is necessary to develop the similarity function to measure the similarity between the collected 


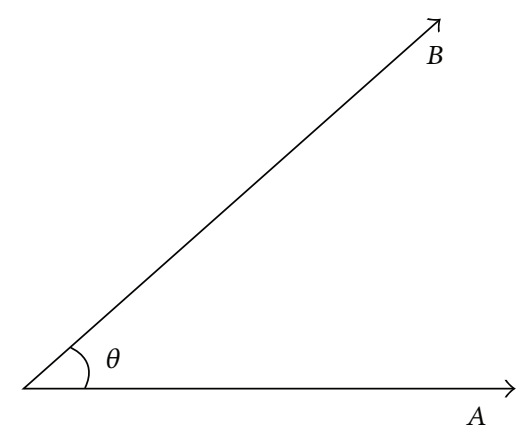

FIGURE 1: Geometric explanation of cosine similarity measurement.

bodies of evidence. The formulation of cosine similarity of vectors $[43,47]$ is defined as follows:

$$
\begin{aligned}
\operatorname{si}_{\cos }(A, B) & =\cos (\theta)=\frac{A \cdot B}{|A||B|} \\
& =\frac{\sum_{i=1}^{n} A_{i} * B_{i}}{\sqrt{\sum_{i=1}^{n}\left(A_{i}\right)^{2}} * \sqrt{\sum_{i=1}^{n}\left(B_{i}\right)^{2}}},
\end{aligned}
$$

where $0 \leq \cos (\theta) \leq 1$.

If angle $\theta$ between $A$ and $B$ is $0^{\circ}$, then their similarity is 1. On the contrary, if the angle between them is $90^{\circ}$, then the similarity is 0 . The geometric explanation of cosine similarity measurement is shown in Figure 1.

\section{The Problem of Existing Conflict Measurements between BPAs}

Example 11. Let $\Theta$ be a frame of discernment with five elements $w_{1}, w_{2}, w_{3}, w_{4}, w_{5}$. We assume the BPAs from three distinct sources, and they are defined as follows:

$$
\begin{aligned}
m_{1}\left(w_{1}\right) & =m_{1}\left(w_{2}\right)=m_{1}\left(w_{3}\right)=m_{1}\left(w_{4}\right)=m_{1}\left(w_{5}\right) \\
& =0.2 \\
m_{2}\left(w_{1}\right) & =m_{2}\left(w_{2}\right)=m_{2}\left(w_{3}\right)=m_{2}\left(w_{4}\right)=m_{2}\left(w_{5}\right) \\
& =0.2 \\
m_{3}\left(w_{1}\right) & =m_{3}\left(w_{2}\right)=m_{3}\left(w_{3}\right)=m_{3}\left(w_{4}\right)=0.25
\end{aligned}
$$

If classical conflict coefficient $k$ is used to measure the degree of conflict among bodies of evidence, we get $k\left(m_{1}, m_{2}\right)=$ $k\left(m_{1}, m_{3}\right)=0.8$, which indicates that the degree of conflict between $m_{1}$ and $m_{2}$ is equal to the degree of conflict between $m_{1}$ and $m_{3}$, and they are both in high conflict. In fact, since $m_{1}$ is identical to $m_{2}$, they are not in conflict. Moreover, the degree of conflict between $m_{1}$ and $m_{3}$ intuitively should be higher than the degree of conflict between $m_{1}$ and $m_{2}$. With Liu's method, we get $c f\left(m_{1}, m_{2}\right)=\left\langle k\left(m_{1}, m_{2}\right)\right.$, $\left.\operatorname{dif} \operatorname{Bet} P\left(m_{1}, m_{2}\right)\right\rangle=\langle 0.8,0\rangle$, which considers that $m_{1}$ and $m_{2}$ have no apparent severe different beliefs and Dempster's combination rule should be used with caution.

This example shows that classical conflict coefficient $k$ cannot measure the degree of conflict between bodies of
TABLE 1: Comparison of $k$ and $d_{j}$ values of five pairs of BPAs in Example 12.

\begin{tabular}{llc}
\hline Pair & $k$ & $d_{j}$ \\
\hline 1st & 0 & 0.7071 \\
2nd & 0 & 0.6325 \\
3rd & 0 & 0.5774 \\
4th & 0 & 0.5345 \\
5th & 0 & 0.5 \\
\hline
\end{tabular}

evidence. Liu's method $c f=\langle k, \operatorname{difBet} P\rangle$ is not good enough to measure the degree of conflict.

Example 12. Let $m_{1}$ and $m_{2}$ be two BPAs from two distinct sources on $\Theta=\left\{w_{1}, w_{2}, \ldots, w_{8}\right\}$. The five pairs of BPAs are shown as follows:

$$
\begin{aligned}
& \text { 1st pair: } m_{1}\left(w_{1}, w_{2}, w_{3}\right)=1, m_{2}\left(w_{1}, w_{2}, w_{4}\right)=1 \text {; } \\
& \text { 2nd pair: } m_{1}\left(w_{1}, w_{2}, w_{3}, w_{4}\right)=1, m_{2}\left(w_{1}, w_{2}, w_{3}, w_{5}\right) \\
& \text { = 1; } \\
& \text { 3rd pair: } m_{1}\left(w_{1}, w_{2}, w_{3}, w_{4}, w_{5}\right)=1, m_{2}\left(w_{1}, w_{2}, w_{3}\right. \text {, } \\
& \left.w_{4}, w_{6}\right)=1 \text {; } \\
& \text { 4th pair: } m_{1}\left(w_{1}, w_{2}, w_{3}, w_{4}, w_{5}, w_{6}\right)=1, m_{2}\left(w_{1}, w_{2}\right. \text {, } \\
& \left.w_{3}, w_{4}, w_{5}, w_{7}\right)=1 ; \\
& \text { 5th pair: } m_{1}\left(w_{1}, w_{2}, w_{3}, w_{4}, w_{5}, w_{6}, w_{7}\right)=1, m_{2}\left(w_{1}\right. \text {, } \\
& \left.w_{2}, w_{3}, w_{4}, w_{5}, w_{6}, w_{8}\right)=1 .
\end{aligned}
$$

The summary of $k$ and $d_{j}$ values of the five pairs is given in Table 1. From the 1st pair of BPAs to the 5 th pair of BPAs, we can know that two BPAs always have only one incompatible element and the number of compatible elements increases from two to six. Intuitively, every pair of BPAs is in certain conflict and the degree of conflict between $m_{1}$ and $m_{2}$ should be lower than the similarity between $m_{1}$ and $m_{2}$. In addition, the degree of conflict should decrease and the similarity should increase from the 1st pair of BPAs to the 5 th pair of BPAs.

As can be seen from Table $1, k=0$ is constant for five pairs of BPAs and it indicates that there is no conflict. Although the Jousselme distance $d_{j}$ decreases from the 1st pair of BPAs to the 5 th pair of BPAs, the value of $d_{j}$ is higher than 0.5 which means that there is high conflict between BPAs. Obviously, both $k$ and $d_{j}$ are counterintuitive.

This example shows that $k$ cannot reveal the conflict between BPAs and $d_{j}$ is not good enough to characterize conflict precisely.

Example 13. Let $m_{1}, m_{2}$ be two BPAs from two distinct sources on frame $\Theta=\left\{w_{1}, w_{2}, \ldots, w_{2 n}\right\}$, such that

$m_{1}$ is

$$
m_{1}\left(w_{1}\right)=m_{1}\left(w_{2}\right)=\cdots=m_{1}\left(w_{n}\right)=\frac{1}{n}
$$

and $m_{2}$ is

$$
m_{2}\left(w_{n+1}\right)=m_{2}\left(w_{n+2}\right)=\cdots=m_{2}\left(w_{2 n}\right)=\frac{1}{n} .
$$




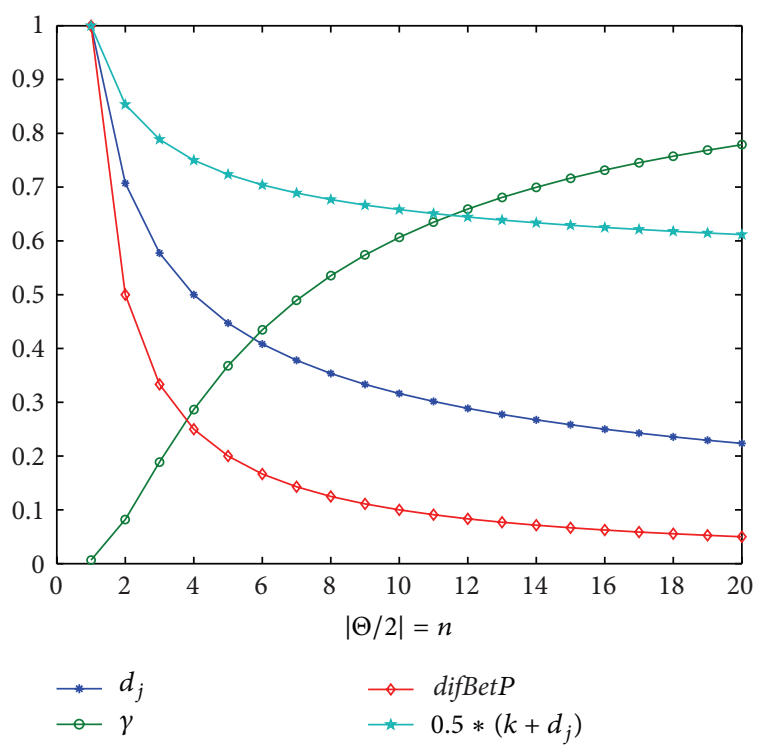

FIGURE 2: Different conflict measurements.

In this example, the two BPAs totally contradict with each other since $m_{1}$ and $m_{2}$ support the different hypothesis. In Song et al's method [49], a new measurement of conflict was presented based on the definition of correlation coefficient. This method is reasonable and effective. But in this example, it is unreasonable. When $n=1$, we get $\operatorname{cor}\left(m_{1}, m_{2}\right)=0.2$. When $n=2$, we get $\operatorname{cor}\left(m_{1}, m_{2}\right)=0.399$. When $n=3$, we get $\operatorname{cor}\left(m_{1}, m_{2}\right)=0.5606$. According to the value of $\operatorname{cor}\left(m_{1}, m_{2}\right)$, it is unreasonable because of the high conflict. But in our method, it is reasonable. Based on (4), we can obtain that classical conflict coefficient $k=1$ for $n=1,2, \ldots, \infty$. The other different measurements of conflict between $m_{1}$ and $m_{2}$ are shown in Figure 2.

As can be seen from Figure 2, we can know that $d_{j}$, $\operatorname{difBet} P$ and $0.5 *\left(k+d_{j}\right)$ are $1, \gamma=0$ when $n=1$, which are consistent with the above analysis. But when $n>1$, they are all against the above intuitive analysis. The values of $\operatorname{dif} \operatorname{Bet} P$ and $d_{j}$ tend toward $0,0.5 *\left(k+d_{j}\right)$ tends toward 0.5 , and $\gamma\left(m_{1}, m_{2}\right)$ tends toward 1 , which indicates that $m_{1}$ and $m_{2}$ are closer and closer with the increase of $n$. The results are counterintuitive and reasonable. Therefore, they cannot be used to measure the conflict of BPAs in this example.

\section{New Similarity of BPAs}

To determine whether there is conflict between bodies of evidence, the similarity between bodies of evidence can be considered. If there is little conflict between bodies of evidence, then the similarity is high. If the two bodies of evidence are in high conflict, then the similarity is low. Therefore the similarity of BPAs can be used to measure the degree of conflict between bodies of evidence.

4.1. Modified Cosine Similarity of Vectors. Cosine similarity in $[43,50]$ is a measurement of similarity between two vectors

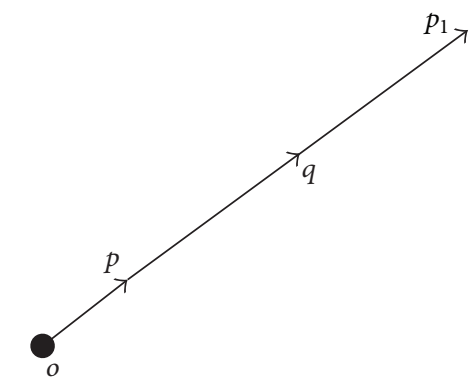

FIGURE 3: Integrated similarity analysis.

of an inner product space that measures the cosine of the angle between them. The angle-based cosine similarity is a direction-based similarity measure. Therefore, it measures the similarity between two vectors only based on the direction but ignoring the impact of the distance of two vectors. In [50], Zhang and Korfhage proposed a new similarity measurement (integrated similarity measurement) based on distance and angle. The integrated similarity measurement is defined as follows:

$$
s=\alpha^{-d} * c^{k}
$$

where $\alpha^{-d}$ is a distance-based similarity measurement [7]. $d$ is the Euclidean distance between vectors $A$ and $B$, and $\alpha$ is a constant whose value is greater than $1 . c^{k}$ is a modifier based on angle. More detailed information can be found in [50].

Although the integrated similarity measurement takes the strengths of both the distance and direction of two vectors into account, there still exist deficiencies. For example, in Figure 3, the angle $\theta$ between vector $o p\left(o p_{1}\right)$ and $o q$ is $0^{\circ}$ and $|o p|=0.1,|p q|=\left|q p_{1}\right|=5$.

According to (22) and the same parameters in [50], we can get the conclusion: $s(o p, o q)=s\left(o p_{1}, o q\right)$. But we think that the similarity between $o p$ and $o q$ should be different from the similarity between $o p_{1}$ and $o q$, since from Figure 3, we can get that $\angle p o q=\angle p_{1} o q=0^{\circ}$ and $|o q-o p|=|p q|=$ $\left|o p_{1}-o q\right|=\left|q p_{1}\right|=5$, but $|o p| /|o q|=0.0197$ is much smaller than $|o q| /\left|o p_{1}\right|=0.505$. Intuitively, it is expected that $|o q|$ is more similar to $\left|o p_{1}\right|$ than to $|o p|$. Therefore, we consider that the similarity between $o p_{1}$ and $o q$ should be higher than the similarity between op and oq.

From the above example, we think that the vector norm (vector magnitude) should be an important factor for the similarity between vectors. The bigger the ratio of vector norm (the smaller vector norm is numerator) is, the higher the similarity between two vectors will be. In order to measure precisely the similarity between two vectors, vector norm should be taken into account besides distance and angle. From the above analysis, the modified cosine similarity based on angle, distance, and vector norm is proposed in Definition 14. 
Definition 14. Let $A=\left[a_{1}, a_{2}, \ldots, a_{n}\right]$ and $B=\left[b_{1}, b_{2}, \ldots, b_{n}\right]$ be two vectors of $R^{n}$. The modified cosine similarity between vectors $A$ and $B$ is defined below:

$$
\begin{aligned}
& \operatorname{SI}(A, B) \\
& = \begin{cases}\frac{1}{2}\left\{\alpha^{-d}+\min \left(\frac{|A|}{|B|}, \frac{|B|}{|A|}\right)\right\} \mathrm{si}_{\cos }(A, B) & A \neq 0, B \neq 0 \\
0 & A=0 \text { or } B=0,\end{cases}
\end{aligned}
$$

where $a^{-d}$ is the distance-based similarity measurement [7].

$d$ is the Euclidean distance between vectors $A$ and $B$, and $\alpha$ is a constant whose value is greater than 1 . The larger the $\alpha$ is, the greater the distance impact on vector similarity will be. $\min (|A| /|B|,|B| /|A|)$ is the minimum of $|A| /|B|$ and $|B| /|A|$. $\mathrm{si}_{\cos }(A, B)$ is the cosine similarity which is defined in (18).

The modified cosine similarity satisfies the following properties:

(1) $\operatorname{SI}(A, B)=\operatorname{SI}(B, A)$ (symmetry);

(2) $0 \leq \operatorname{SI}(A, B) \leq 1$ (nonnegativity);

(3) when $A \neq 0$ and $B \neq 0, \operatorname{SI}(A, B)=1 \Leftrightarrow A=B$;

(4) when $A \neq 0$ and $B \neq 0, \operatorname{SI}(A, B)=0 \Leftrightarrow A \perp B$;

Proof of Property (1). Based on (18) and (23), it is easy to get $\operatorname{SI}(A, B)=\operatorname{SI}(B, A)$.

Proof of Property (2). If $A \neq 0$ and $B \neq 0$, then $d \geq 0$, $0<\min (|A| /|B|,|B| /|A|) \leq 1$, and $0 \leq \operatorname{si}_{\cos } \leq 1$. Hence $0<$ $a^{-d} \leq 1$ when $a \geq 1$. Then, based on (23), we can obtain $0 \leq$ $\mathrm{SI}(A, B) \leq 1$. In addition, if $A=0$ or $B=0, \operatorname{SI}(A, B)=0$. In summary, $0 \leq \operatorname{SI}(A, B) \leq 1$.

Proof of Property (3). When $A \neq 0$ and $B \neq 0$, if $A=B$, namely, $d=0,|A|=|B|>0$ and $\theta=0$, we can get the following formulas based on (18) and (23):

$$
\begin{aligned}
\alpha^{-d} & =1 \\
\min \left(\frac{|A|}{|B|}, \frac{|B|}{|A|}\right) & =1 \\
\operatorname{si}_{\cos } & =1 .
\end{aligned}
$$

It is obvious that $\operatorname{SI}(A, B)=1$. When $A \neq 0$ and $B \neq 0$, we can see that $0<\alpha^{-d} \leq 1,0<\min (|A| /|B|,|B| /|A|) \leq 1$, and $0 \leq \mathrm{si}_{\cos } \leq 1$. Hence, if $\operatorname{SI}(A, B)=1$, we can obtain $\alpha^{-d}=$ $\min (|A| /|B|,|B| /|A|)=\operatorname{si}_{\cos }=1$; namely, $d=0,|A|=|B|$, and $\theta=0$; therefore $A=B$. In summary, when $A \neq 0$ and $B \neq 0, \operatorname{SI}(A, B)=1 \Leftrightarrow A=B$.

Proof of Property (4). If $A \neq 0$ and $B \neq 0$, then $\min (|A| /|B|,|B| /|A|)>0$. Hence, based on $(23)$, if $\operatorname{SI}(A, B)=$ 0 , then $\mathrm{si}_{\mathrm{cos}}=0$. Therefore, based (18), we can get $A \perp B$.

If $A \perp B$, we can obtain $\mathrm{si}_{\text {cos }}=0$ based on (18). Furthermore we can obtain $\operatorname{SI}(A, B)=0$ based on (23). In summary, when $A \neq 0$ and $B \neq 0, \operatorname{SI}(A, B)=0 \Leftrightarrow A \perp B$.
4.2. New Similarity of BPAs Based on the Modified Cosine Similarity. Under the frame of discernment $\Theta=\left\{w_{1}, w_{2}, \ldots\right.$, $\left.w_{N}\right\}$, there are two evidence sources $E_{1}$ and $E_{2}$. Let $m_{1}$ and $m_{2}$ be the BPAs, respectively. $m_{1}$ and $m_{2}$ can be expressed, respectively, as $\left[\operatorname{Bel}_{1}\left(w_{j}\right), \mathrm{Pl}_{1}\left(w_{j}\right)\right], j=1, \ldots, N$, and $\left[\operatorname{Bel}_{2}\left(w_{j}\right), \mathrm{Pl}_{2}\left(w_{j}\right)\right], j=1, \ldots, N$, where $w_{j}, j=1, \ldots, N$ are the singleton subsets [6].

According to the above confidence intervals, BPAs are in the form of two vectors on the singleton subsets. The two vectors are expressed, respectively, below:

$$
\begin{aligned}
& \operatorname{Bel}_{i}=\left[\operatorname{Bel}_{i}\left(w_{1}\right), \operatorname{Bel}_{i}\left(w_{2}\right), \ldots, \operatorname{Bel}_{i}\left(w_{N}\right)\right], \\
& i=1,2 \\
& \mathrm{Pl}_{i}=\left[\operatorname{Pl}_{i}\left(w_{1}\right), \mathrm{Pl}_{i}\left(w_{2}\right), \ldots, \mathrm{Pl}_{i}\left(w_{N}\right)\right], \quad i=1,2 .
\end{aligned}
$$

Then we can calculate the belief function vector similarity $\mathrm{SI}\left(\mathrm{Bel}_{1}, \mathrm{Bel}_{2}\right)$ and the plausibility function vector similarity $\mathrm{SI}\left(\mathrm{Pl}_{1}, \mathrm{Pl}_{2}\right)$ based on the modified cosine similarity.

Finally, the new similarity of BPAs is defined below:

$$
\mathrm{SI}_{\mathrm{BPA}}=(1-\beta) * \mathrm{SI}\left(\mathrm{Bel}_{1}, \mathrm{Bel}_{2}\right)+\beta * \mathrm{SI}\left(\mathrm{Pl}_{1}, \mathrm{Pl}_{2}\right)
$$

with

$$
0 \leq \beta \leq 1,
$$

where $\beta$ is the total uncertainty of BPAs, which is defined as

$$
\beta=\frac{\sum_{i=1}^{2} \sum_{j=1}^{N}\left(\mathrm{Pl}_{i}\left(w_{j}\right)-\operatorname{Bel}_{i}\left(w_{j}\right)\right)}{\sum_{i=1}^{2} \sum_{j=1}^{N}\left(\mathrm{Pl}_{i}\left(w_{j}\right)\right)} .
$$

Because of $\operatorname{Pl}_{i}\left(w_{j}\right) \geq \operatorname{Bel}_{i}\left(w_{j}\right)$ and $\beta \geq 0$, we get that if $\operatorname{Pl}_{i}\left(w_{j}\right)=\operatorname{Bel}_{i}\left(w_{j}\right)$, then $\beta$ will obtain the minimum value 0 ; if $\operatorname{Bel}_{i}\left(w_{j}\right)=0$, then $\beta$ will obtain the maximum value 1 .

The belief $\operatorname{Bel}\left(w_{i}\right)$ in the confidence interval represents the minimum trust that explicitly supports $w_{i}$, and the plausibility $\mathrm{Pl}\left(w_{i}\right)$ expresses the potential support for $w_{i} \cdot \operatorname{Pl}\left(w_{i}\right)-$ $\operatorname{Bel}\left(w_{i}\right)$ indicates the uncertainty of $w_{i}$. Obviously, the larger the uncertainty $\beta$ is, the higher the influence on the similarity of BPA will be, but the influence of belief function similarity on the similarity of BPA should be lower. The new similarity of BPAs $\mathrm{SI}_{\mathrm{BPA}}$ satisfies the following properties:

(1) $m_{1}=m_{2} \Rightarrow \operatorname{SI}_{\mathrm{BPA}}\left(m_{1}, m_{2}\right)=1$;

(2) $\mathrm{SI}_{\mathrm{BPA}}\left(m_{1}, m_{2}\right)=\mathrm{SI}_{\mathrm{BPA}}\left(m_{2}, m_{1}\right)$;

(3) $0 \leq \mathrm{SI}_{\mathrm{BPA}}\left(m_{1}, m_{2}\right) \leq 1$;

(4) $\mathrm{SI}_{\mathrm{BPA}}\left(m_{1}, m_{2}\right)=0$, if and only if $m_{1}$ and $m_{2}$ have no compatible element.

Some proofs are detailed as follows.

Proof of Property (3). Based on (23), (25), and (28), we can obtain that

$$
\begin{aligned}
& 0 \leq \beta \leq 1 \\
& 0 \leq 1-\beta \leq 1 \\
& 0 \leq \mathrm{SI}\left(\mathrm{Bel}_{1}, \mathrm{Bel}_{2}\right) \leq 1 \\
& 0 \leq \mathrm{SI}\left(\mathrm{Pl}_{1}, \mathrm{Pl}_{2}\right) \leq 1 .
\end{aligned}
$$


So, we get $\mathrm{SI}_{\mathrm{BPA}}\left(m_{1}, m_{2}\right) \geq 0$ based on (26). Moreover, if $\mathrm{SI}\left(\mathrm{Bel}_{1}, \mathrm{Bel}_{2}\right)=\mathrm{SI}\left(\mathrm{Pl}_{1}, \mathrm{Pl}_{2}\right)=1$, then $\mathrm{SI}_{\mathrm{BPA}}\left(m_{1}, m_{2}\right)$ will get the maximum value 1 when $\beta \in[0,1]$. In summary, $0 \leq \mathrm{SI}_{\mathrm{BPA}}\left(m_{1}, m_{2}\right) \leq 1$.

Proof of Property (4). Assuming that $\beta=0$, according to (28), we can obtain $\operatorname{Pl}_{i}\left(w_{j}\right)=\operatorname{Bel}_{i}\left(w_{j}\right), i=1,2, j=1,2, \ldots, N$; then $\mathrm{SI}\left(\mathrm{Bel}_{1}, \mathrm{Bel}_{2}\right)=\mathrm{SI}\left(\mathrm{Pl}_{1}, \mathrm{Pl}_{2}\right)$. Hence, based on (26), we can get

$$
\begin{aligned}
\mathrm{SI}_{\mathrm{BPA}} & =(1-\beta) * \mathrm{SI}\left(\mathrm{Bel}_{1}, \mathrm{Bel}_{2}\right)+\beta * \mathrm{SI}\left(\mathrm{Pl}_{1}, \mathrm{Pl}_{2}\right) \\
& =\mathrm{SI}\left(\mathrm{Bel}_{1}, \mathrm{Bel}_{2}\right) .
\end{aligned}
$$

If $\mathrm{SI}_{\mathrm{BPA}}\left(m_{1}, m_{2}\right)=0$, then $\mathrm{SI}\left(\mathrm{Bel}_{1}, \mathrm{Bel}_{2}\right)=0$; namely, $\mathrm{SI}\left(\mathrm{Pl}_{1}\right.$, $\left.\mathrm{Pl}_{2}\right)=0 . \mathrm{SI}\left(\mathrm{Pl}_{1}, \mathrm{Pl}_{2}\right)=0$ means that $m_{1}$ and $m_{2}$ have no compatible element.

Assuming that $\beta=1$, if $\mathrm{SI}_{\mathrm{BPA}}\left(m_{1}, m_{2}\right)=0$, we can easily obtain $\mathrm{SI}\left(\mathrm{Pl}_{1}, \mathrm{Pl}_{2}\right)=0$ based on (26), which means that $m_{1}$ and $m_{2}$ have no compatible element.

If $0<\beta<1$, based on (26), we can easily see that

$$
\begin{aligned}
\mathrm{SI}_{\mathrm{BPA}}\left(m_{1}, m_{2}\right) & =0 \Longrightarrow \mathrm{SI}\left(\mathrm{Pl}_{1}, \mathrm{Pl}_{2}\right)=\mathrm{SI}\left(\mathrm{Bel}_{1}, \mathrm{Bel}_{2}\right) \\
& =0
\end{aligned}
$$

which means that $m_{1}$ and $m_{2}$ have no compatible element.

If $m_{1}$ and $m_{2}$ are not compatible, it is not difficult to obtain $\mathrm{SI}\left(\mathrm{Pl}_{1}, \mathrm{Pl}_{2}\right)=\mathrm{SI}\left(\mathrm{Bel}_{1}, \mathrm{Bel}_{2}\right)=0$; then we could obtain $\mathrm{SI}_{\mathrm{BPA}}\left(m_{1}, m_{2}\right)=0$. In summary, if and only if $m_{1}$ and $m_{2}$ have no compatible elements, $\operatorname{SI}_{\mathrm{BPA}}\left(m_{1}, m_{2}\right)=0$.

The establishment of the above four properties shows that the new similarity $\mathrm{SI}_{\mathrm{BPA}}$ is the characteristic metrics of similarity of evidence and is consistent with the distribution of bodies of evidence $m_{1}$ and $m_{2}$. Therefore, $\mathrm{SI}_{\mathrm{BPA}}$ can be used to measure the conflict (similarity) between bodies of evidence $m_{1}$ and $m_{2}$. Since the modified cosine similarity SI takes the impact of the ratio of vector norm into account, SI overcomes the deficiency of the integrated similarity measurement in [50]. Moreover, the new similarity of BPA based on the modified cosine similarity takes the impact of belief function, plausibility function, and uncertainty $\beta$ into account, so $\mathrm{SI}_{\mathrm{BPA}}$ can effectively measure the degree of conflict between bodies of evidence.

\section{Example and Analysis}

In this section, four examples are presented to illustrate the effectiveness of the proposed similarity of BPAs.

Example 15. Considering the three BPAs which we defined in Example 11. With the proposed similarity of $\mathrm{BPAs} \mathrm{SI}_{\mathrm{BPA}}$, the following results are obtained:

$$
\begin{aligned}
1-\mathrm{SI}_{\mathrm{BPA}}\left(m_{1}, m_{2}\right) & =0<1-\mathrm{SI}_{\mathrm{BPA}}\left(m_{1}, m_{3}\right) \\
& =0.1707
\end{aligned}
$$

which indicates that $m_{1}$ and $m_{2}$ completely agree with each other, and the degree of conflict between $m_{1}$ and $m_{2}$ is lower than the degree of conflict between $m_{1}$ and $m_{3}$. The result is consistent with intuitive analysis.
TABLE 2: $\mathrm{SI}_{\text {BPA }}$ values of five pairs of BPAs in Example 12.

\begin{tabular}{lccccc}
\hline Pair & 1st & 2nd & 3rd & 4th & 5th \\
\hline $\mathrm{SI}_{\mathrm{BPA}}$ & 0.5909 & 0.6648 & 0.7091 & 0.7386 & 0.7579 \\
\hline
\end{tabular}

Example 16. Consider the five pairs of BPAs in Example 12. With the proposed similarity of $\mathrm{BPAs} \mathrm{SI}_{\mathrm{BPA}}$, the results are listed in Table 2.

From Table 2, the value of $\mathrm{SI}_{\mathrm{BPA}}$ increases from 0.5909 to 0.7597 and the similarity is higher than others. The result is consistent with intuitive analysis.

Example 17. Consider the two BPAs which we defined in Example 13. With the proposed similarity of BPAs, we obtain $\mathrm{SI}_{\mathrm{BPA}}\left(m_{1}, m_{2}\right)=0$ for $n=1, \ldots, 20$. It indicates that $m_{1}$ and $m_{2}$ are completely different. That is to say, they are in total conflict which is consistent with the analysis in Example 13.

Example 18. Let $\Theta$ be a frame of discernment with 20 elements (or any number of elements that is predefined). For convenience, we use 1,2 , and so forth to denote $w_{1}, w_{2}$, and so forth in the frame.

The first BPA, $m_{1}$, is defined as follows:

$$
\begin{aligned}
m_{1}(2,3,4) & =0.05 \\
m_{1}(5) & =0.05 \\
m_{1}(\Theta) & =0.1, \\
m_{1}(A) & =0.8
\end{aligned}
$$

where $A$ is a subset of $\Theta$.

And the second BPA, $m_{2}$, is defined as follows:

$$
m_{2}(1,2,3,4,5)=1 \text {. }
$$

There are 20 cases where subset $A$ increases by one more element at a time, starting from case 1 with $A=\{1\}$ and ending with case 20 , where $A=\{\Theta\}$ as shown in Table 3. The comparison of the aforementioned conflict measurements between $m_{1}$ and $m_{2}$ for these 20 cases is detailed in Table 3 and graphically illustrated in Figure 4.

In this example, the conflict should be lower from case 1 to case 5 , since the supported hypothesis of $m_{1}$ and $m_{2}$ gets more and more similar. In case $5, A=\{1,2,3,4,5\}$ is identical to hypothesis of $m_{2}$; hence the degree of conflict between $m_{1}$ and $m_{2}$ should obtain the minimum value. The conflict should increase from case 5 to case 20 , since $m_{1}$ becomes more and more uncertain.

From Figure 4, we know that conflict measurement $d_{j}$, $0.5\left(k+d_{j}\right)$ and $\operatorname{difBetP}$ go up and down consistently when the size of $A$ changes. Although $0.5\left(k+d_{j}\right)$ and $d_{j}$ present a similar behavior, the value of $0.5\left(k+d_{j}\right)$ is always smaller than the corresponding $d_{j}$ values. So, we get the conclusion that $0.5\left(k+d_{j}\right)$ is not better than $d_{j}$ to measure conflict among bodies of evidence. $\operatorname{difBet} P<0.25$ from case 2 to case 20 and its values even decrease from case 6 to case 8 which is abnormal. Furthermore, $k=0$ indicates that $m_{1}$ and $m_{2}$ have 
TABLE 3: Comparison of different conflict measurements.

\begin{tabular}{|c|c|c|c|c|c|c|}
\hline Case & $k$ & $\operatorname{difBet} P$ & $d_{j}$ & $\gamma$ & $0.5 *\left(k+d_{j}\right)$ & $\mathrm{SI}_{\mathrm{BPA}}$ \\
\hline$A=\{1\}$ & 0 & 0.6050 & 0.7856 & 0.3313 & 0.3928 & 0.3468 \\
\hline$A=\{1,2\}$ & 0 & 0.2270 & 0.6864 & 0.6422 & 0.3432 & 0.5074 \\
\hline$A=\{1,2,3\}$ & 0 & 0.1783 & 0.5702 & 0.8579 & 0.2851 & 0.6348 \\
\hline$A=\{1, \ldots, 4\}$ & 0 & 0.1450 & 0.4232 & 0.9290 & 0.2116 & 0.7685 \\
\hline$A=\{1, \ldots, 5\}$ & 0 & 0.0350 & 0.1309 & 0.9308 & 0.0654 & 0.9219 \\
\hline$A=\{1, \ldots, 6\}$ & 0 & 0.1330 & 0.3879 & 0.8913 & 0.1940 & 0.8132 \\
\hline$A=\{1, \ldots, 7\}$ & 0 & 0.1143 & 0.5025 & 0.7014 & 0.2512 & 0.7101 \\
\hline$A=\{1, \ldots, 8\}$ & 0 & 0.1000 & 0.5702 & 0.6773 & 0.2851 & 0.6320 \\
\hline$A=\{1, \ldots, 9\}$ & 0 & 0.1060 & 0.6184 & 0.6436 & 0.3092 & 0.5702 \\
\hline$A=\{1, \ldots, 10\}$ & 0 & 0.1150 & 0.6551 & 0.6066 & 0.3276 & 0.5199 \\
\hline$A=\{1, \ldots, 11\}$ & 0 & 0.1223 & 0.6841 & 0.5698 & 0.3421 & 0.4780 \\
\hline$A=\{1, \ldots, 12\}$ & 0 & 0.1283 & 0.7079 & 0.5351 & 0.3539 & 0.4424 \\
\hline$A=\{1, \ldots, 13\}$ & 0 & 0.1335 & 0.7278 & 0.5031 & 0.3639 & 0.4119 \\
\hline$A=\{1, \ldots, 14\}$ & 0 & 0.1379 & 0.7449 & 0.4740 & 0.3725 & 0.3853 \\
\hline$A=\{1, \ldots, 15\}$ & 0 & 0.1417 & 0.7597 & 0.4477 & 0.3799 & 0.3620 \\
\hline$A=\{1, \ldots, 16\}$ & 0 & 0.1450 & 0.7727 & 0.4241 & 0.3864 & 0.3414 \\
\hline$A=\{1, \ldots, 17\}$ & 0 & 0.1479 & 0.7844 & 0.4027 & 0.3922 & 0.3230 \\
\hline$A=\{1, \ldots, 18\}$ & 0 & 0.1506 & 0.7949 & 0.3835 & 0.3975 & 0.3064 \\
\hline$A=\{1, \ldots, 19\}$ & 0 & 0.1529 & 0.8044 & 0.3661 & 0.4022 & 0.2915 \\
\hline$A=\{1, \ldots, 20\}$ & 0 & 0.1550 & 0.8131 & 0.3627 & 0.4066 & 0.2779 \\
\hline
\end{tabular}

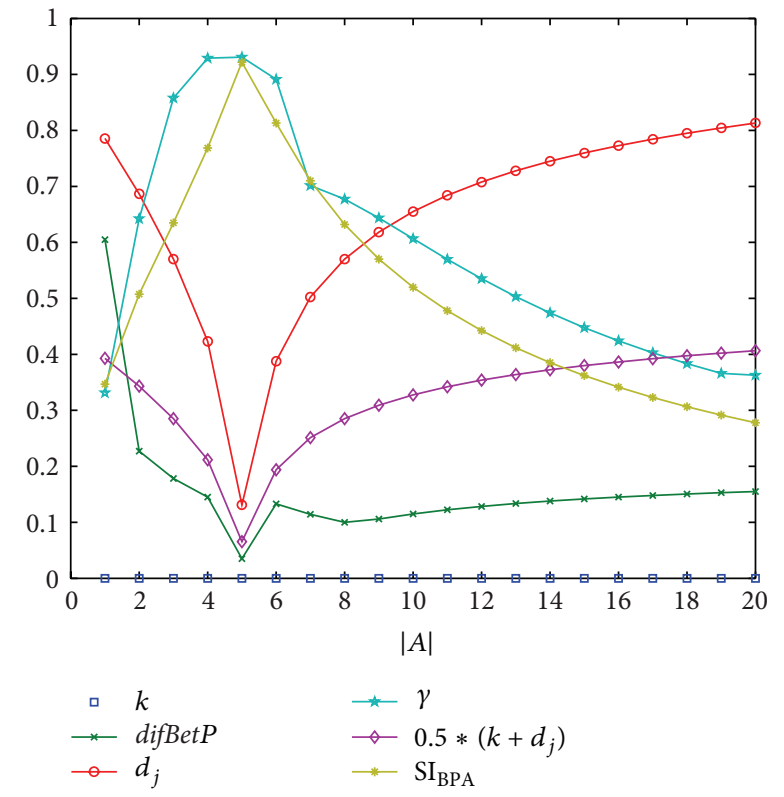

FIGURE 4: Comparison of different conflict measurements between $m_{1}$ and $m_{2}$.

no conflict which is opposite to our analysis. Because both $k$ and $\operatorname{dif} B e t P$ are low, Liu's method $\langle k, \operatorname{dif} B e t P\rangle$ indicates that there is little contradiction between $m_{1}$ and $m_{2}$ from case 2 to case 20 . Therefore $\langle k$, $\operatorname{difBet} P\rangle$ cannot reflect the conflict changes when the size of $A$ changes. Whereas $\gamma$ and SI $_{\text {BPA }}$ are used to measure the similarity among BPAs. The bigger the $\gamma$ and $\mathrm{SI}_{\mathrm{BPA}}$ is, the lower the degree of conflict will be. The curves of $d_{\mathrm{BPA}}, \gamma$, and $\mathrm{SI}_{\mathrm{BPA}}$ are consistent with the above analysis; therefore we think that $\mathrm{SI}_{\mathrm{BPA}}$ can also be used to characterize the degree of conflict.

According to the above four examples, we get a conclusion that the new similarity of BPAs $\mathrm{SI}_{\mathrm{BPA}}$ can efficiently characterize the degree of conflict between BPAs.

\section{A New Combination Method and Application}

In this section, we proposed a new combination method based on the new similarity of BPAs. If there are $n$ bodies of evidence (BOEs) $m_{i}, i=1, \ldots, n$, the calculation process is listed as follows.

Step 1. Calculate the similarity between the bodies of evidence; then construct a similarity measure matrix (SMM), which gives us insight into the agreement between evidence, where $\mathrm{SI}_{\mathrm{BPA}}(i j)$ is the similarity between $m_{i}$ and $m_{j}$ :

SMM

$$
=\left(\begin{array}{ccccc}
\mathrm{SI}_{\mathrm{BPA}}(11) & \cdots & \mathrm{SI}_{\mathrm{BPA}}(1 i) & \cdots & \mathrm{SI}_{\mathrm{BPA}}(1 n) \\
\vdots & \vdots & \vdots & \vdots & \vdots \\
\mathrm{SI}_{\mathrm{BPA}}(i 1) & \cdots & \mathrm{SI}_{\mathrm{BPA}}(i i) & \cdots & \mathrm{SI}_{\mathrm{BPA}}(i n) \\
\vdots & \vdots & \vdots & \vdots & \vdots \\
\mathrm{SI}_{\mathrm{BPA}}(n 1) & \cdots & \mathrm{SI}_{\mathrm{BPA}}(n i) & \cdots & \mathrm{SI}_{\mathrm{BPA}}(n n)
\end{array}\right) .
$$


Step 2. The credibility of body of evidence $m_{i}$ is defined as follows:

$$
\operatorname{Sup}\left(m_{i}\right)=\sum_{\substack{j=1 \\ j \neq i}}^{n} \operatorname{SI}_{\mathrm{BPA}}(i j), \quad i=1, \ldots, n .
$$

We know that the lower the distance between the bodies of evidence is, the higher the similarity is.

Step 3. The credibility degree $\mathrm{Crd}_{i}$ of the body of evidence $m_{i}$ is defined as follows:

$$
\operatorname{Crd}_{i}=\frac{\operatorname{Sup}\left(m_{i}\right)}{\sum_{i=1}^{n} \operatorname{Sup}\left(m_{i}\right)} .
$$

Step 4. The weighted average evidence MAE is given by the following formula:

$$
\operatorname{MAE}(m)=\sum_{i=1}^{n}\left(\operatorname{Crd}_{i} \times m_{i}\right)
$$

Step 5. When the number of original bodies of evidence is $n$, we should combine the MAE for $(n-1)$ times. Then the result can be calculated. We can effectively deal with the information fusion problems in high-conflict evidence by this method.

In general, if a body of evidence is supported by others greatly, the evidence should be more important and has more effect on the final combination result [44]. This method has a faster convergence speed and improves the reliability and rationality when the evidence is contradictory. An example of target recognition is presented to show the validity of the proposed method.

Example 19. There is a target that is detected in combat air domain and the target is identified as enemy by military Identification Friend or Foe. Five different sensors such as airborne early warning radar (AEW radar) and electronic warfare support measure (EMS) detect the air targets, and the frame of discernment is $\Theta=\{A, B, C\}$. At a certain moment, five bodies of evidence have been collected from these five different sensors. The BPAs $m_{1}, m_{2}, m_{3}, m_{4}, m_{5}$ are given as follows:

$m_{1}$ :

$$
\begin{aligned}
& m_{1}(A)=0.41, \\
& m_{1}(B)=0.29, \\
& m_{1}(C)=0.30 ;
\end{aligned}
$$

$m_{2}$ :

$$
\begin{aligned}
& m_{2}(A)=0.00, \\
& m_{2}(B)=0.90, \\
& m_{2}(C)=0.10
\end{aligned}
$$

$m_{3}:$

$$
\begin{aligned}
m_{3}(A) & =0.58, \\
m_{3}(B) & =0.07, \\
m_{3}(A C) & =0.35 ;
\end{aligned}
$$

$m_{4}:$

$$
\begin{aligned}
m_{4}(A) & =0.55, \\
m_{4}(B) & =0.10, \\
m_{4}(A C) & =0.35 ;
\end{aligned}
$$

$m_{5}:$

$$
\begin{gathered}
m_{5}(A)=0.60, \\
m_{5}(B)=0.10, \\
m_{5}(A C)=0.30 .
\end{gathered}
$$

In this example, it is clear that $m_{2}$ is in high conflict with others. The results by different combination rules are shown in Table 4.

As can be seen from Table 4, Dempster's rule produces counterintuitive result, though more bodies of evidence collected later support target A. Although Murphy's method, Deng's method, and the proposed method can recognize the true target, the performance of convergence of the proposed method is better than others. The main reason is that the proposed method has better performance in conflict management. By making use of the new similarity of BPAs among bodies of evidence, the modified average approach decreases the weight of the "bad" evidence, so the "bad" evidence has less effect on the final results.

In conclusion, the weighted average method based on the proposed similarity measure $\mathrm{SI}_{\mathrm{BPA}}$ can solve the problem of combination with high-conflict evidence. It illustrates the efficiency and the validity of the new method to deal with the high-conflict evidence.

\section{Conclusion}

In the actual battle field, due to the harsh environment and human interference, the high conflict often exists among the sensor reports. In order to solve the problem of conflicting information fusion, the existing methods pay more attention to the evidence combination algorithm while ignoring the study of conflict itself. Are there any other parameters or factors that can reflect the conflict between bodies of evidence? Is there a better conflict model that can comprehensively describe the conflict between bodies of evidence? These issues are the basis for resolving the fusion of conflicting information and are very worthy of research.

In this paper, a new similarity of BPAs is proposed to measure the degree of conflict, which is constituted by the belief function and plausibility function in evidence theory. By expressing the BPAs in the form of vectors on singleton 
TABLE 4: Results of different combination rules of evidence with one bad evidence.

\begin{tabular}{|c|c|c|c|c|c|c|}
\hline BPAs & Method & $m(A)$ & $m(B)$ & $m(C)$ & $m(A C)$ & Results \\
\hline \multirow{4}{*}{$m_{1}, m_{2}, m_{3}$} & Dempster [7] & 0 & 0.6350 & 0.3650 & 0 & $B$ \\
\hline & Murphy [37] & 0.4939 & 0.4180 & 0.0792 & 0.0090 & $A$ \\
\hline & Yong et al. [44] & 0.4974 & 0.4054 & 0.0888 & 0.0084 & $A$ \\
\hline & Proposed method & 0.5761 & 0.3229 & 0.0921 & 0.0089 & $A$ \\
\hline \multirow{4}{*}{$m_{1}, m_{2}, m_{3}, m_{4}$} & Dempster [7] & 0 & 0.3321 & 0.6679 & 0 & $C$ \\
\hline & Murphy [37] & 0.8362 & 0.1147 & 0.0410 & 0.0081 & $A$ \\
\hline & Yong et al. [44] & 0.9089 & 0.0444 & 0.0379 & 0.0089 & $A$ \\
\hline & Proposed method & 0.9186 & 0.0348 & 0.0379 & 0.0087 & $A$ \\
\hline \multirow{4}{*}{$m_{1}, m_{2}, m_{3}, m_{4}, m_{5}$} & Dempster [7] & 0 & 0.1422 & 0.8578 & 0 & $C$ \\
\hline & Murphy [37] & 0.9620 & 0.0210 & 0.0138 & 0.0032 & $A$ \\
\hline & Yong et al. [44] & 0.9820 & 0.0039 & 0.0107 & 0.0034 & $A$ \\
\hline & Proposed method & 0.9861 & 0.0010 & 0.0095 & 0.0034 & $A$ \\
\hline
\end{tabular}

subsets and calculating two similarities based on the modified cosine similarity, a reasonable measurement of similarity (conflict) among bodies of evidence can be achieved. The similarity can meet the basic nature of requiring for similarity. If two bodies of evidence are consistent, the two bodies of evidence support the same hypothesis.

Therefore, the proposed similarity of BPAs should be high; on the contrary, the degree of conflict is low. If the proposed similarity is low, we consider that the two bodies of evidence are in high conflict. The lower the similarity is, the higher the conflict is. Hence, the proposed similarity can be used to measure the degree of conflict. The example of target recognition shows that the similarity of BPAs could be used in sensor data fusion. In the later research, the similarity will be applied as a constraint condition in decision making based on BPAs.

\section{Competing Interests}

The authors declare that there is no conflict of interests regarding the publication of this paper.

\section{Authors' Contributions}

Wen Jiang and Xiyun Qin contributed equally to this work.

\section{Acknowledgments}

The work is partially supported by the National Natural Science Foundation of China (Grant no. 60904099), the Natural Science Basic Research Plan in Shaanxi Province of China (Program no. 2016JM6018), and the Seed Foundation of Innovation and Creation for Graduate Students in Northwestern Polytechnical University (Program no. Z2016122).

\section{References}

[1] L. A. Zadeh, "Fuzzy sets," Information and Control, vol. 8, no. 3, pp. 338-353, 1965.

[2] W. Jiang, Y. Luo, X.-Y. Qin, and J. Zhan, "An improved method to rank generalized fuzzy numbers with different left heights and right heights," Journal of Intelligent \& Fuzzy Systems, vol. 28, no. 5, pp. 2343-2355, 2015.

[3] S.-B. Tsai, M.-F. Chien, Y. Xue et al., "Using the fuzzy DEMATEL to determine environmental performance: a case of printed circuit board industry in Taiwan," PLoS ONE, vol. 10, no. 6, article e0129153, 2015.

[4] H.-C. Liu, J.-X. You, X.-Y. You, and M.-M. Shan, "A novel approach for failure mode and effects analysis using combination weighting and fuzzy VIKOR method," Applied Soft Computing, vol. 28, pp. 579-588, 2015.

[5] A. Mardani, A. Jusoh, and E. K. Zavadskas, "Fuzzy multiple criteria decision-making techniques and applicationstwo decades review from 1994 to 2014," Expert Systems with Applications, vol. 42, no. 8, pp. 4126-4148, 2015.

[6] G. Shafer, A Mathematical Theory of Evidence, Princeton University Press, Princeton, NJ, USA, 1976.

[7] A. P. Dempster, "Upper and lower probabilities induced by a multivalued mapping," The Annals of Mathematical Statistics, vol. 38, no. 2, pp. 325-339, 1967.

[8] Y. Deng, "Generalized evidence theory," Applied Intelligence, vol. 43, no. 3, pp. 530-543, 2015.

[9] W. Jiang, Y. Yang, Y. Luo, and X. Qin, "Determining basic probability assignment based on the improved similarity measures of generalized fuzzy numbers," International Journal of Computers Communications \& Control, vol. 10, no. 3, pp. 333-347, 2015.

[10] B. Walczak and D. L. Massart, "Rough sets theory," Chemometrics and Intelligent Laboratory Systems, vol. 47, no. 1, pp. 1-16, 1999.

[11] S. Greco, B. Matarazzo, and R. Slowinski, "Rough sets theory for multicriteria decision analysis," European Journal of Operational Research, vol. 129, no. 1, pp. 1-47, 2001.

[12] E. K. Zavadskas, J. Antucheviciene, S. H. R. Hajiagha, and S. S. Hashemi, "The interval-valued intuitionistic fuzzy Multimoora method for group decision making in engineering," Mathematical Problems in Engineering, vol. 2015, Article ID 560690, 13 pages, 2015.

[13] L. A. Zadeh, "A note on Z-numbers," Information Sciences, vol. 181, no. 14, pp. 2923-2932, 2011.

[14] B. Kang, D. Wei, Y. Li, and Y. Deng, "A method of converting Znumber to classical fuzzy number," Journal of Information and Computational Science, vol. 9, no. 3, pp. 703-709, 2012.

[15] D. Mohamad, S. A. Shaharani, and N. H. Kamis, "A Z-numberbased decision making procedure with ranking fuzzy numbers 
method," in Proceedings of the 3rd International Conference on Quantitative Sciences and its Applications, vol. 1635 of AIP Conference Proceedings, pp. 160-166, Langkawi, Malaysia, August 2014.

[16] A. S. A. Bakar and A. Gegov, "Multi-layer decision methodology for ranking z-numbers," International Journal of Computational Intelligence Systems, vol. 8, no. 2, pp. 395-406, 2014.

[17] X. Deng and Y. Deng, "D numbers theory: a generalization of dempster-shafer theory," http://arxiv.org/abs/1402.3490 .

[18] X. Deng, Y. Hu, Y. Deng, and S. Mahadevan, "Environmental impact assessment based on D numbers," Expert Systems with Applications, vol. 41, no. 2, pp. 635-643, 2014.

[19] Y. Deng, Y. Liu, and D. Zhou, "An improved genetic algorithm with initial population strategy for symmetric TSP," Mathematical Problems in Engineering, vol. 2015, Article ID 212794, 6 pages, 2015.

[20] Y. Deng, "A threat assessment model under uncertain environment," Mathematical Problems in Engineering, vol. 2015, Article ID 878024, 12 pages, 2015.

[21] J. Kacprzyk and R. R. Yager, "Using fuzzy logic with linguistic quantifiers in mul-tiobjective decision making and optimization: a step towards more human-consistent models," Theory \& Decision Library, vol. 6, pp. 331-350, 1990.

[22] C. Carlsson and R. Fullr, Fuzzy Reasoning in Decision Making and Optimization, Physica, Heidelberg, Germany, 2002.

[23] W.-B. Du, Y. Gao, C. Liu, Z. Zheng, and Z. Wang, "Adequate is better: particle swarm optimization with limited-information," Applied Mathematics and Computation, vol. 268, pp. 832-838, 2015.

[24] W. Jiang, B. Wei, C. Xie, and D. Zhou, "An evidential sensor fusion method in fault diagnosis," Advances in Mechanical Engineering, vol. 8, no. 3, pp. 1-7, 2016.

[25] Y. Deng, S. Mahadevan, and D. Zhou, "Vulnerability assessment of physical protection systems: a bio-inspired approach," International Journal of Unconventional Computing, vol. 11, no. 3-4, pp. 227-243, 2015.

[26] W. Jiang, C. Xie, B. Wei, and D. Zhou, "A modified method for risk evaluation in failure modes and effects analysis of aircraft turbine rotor blades," Advances in Mechanical Engineering, vol. 8, no. 4, pp. 1-16, 2016.

[27] P. Smets and R. Kennes, "The transferable belief model," Artificial Intelligence, vol. 66, no. 2, pp. 191-234, 1994.

[28] S. S. Rao and K. K. Annamdas, "A comparative study of evidence theories in the modeling, analysis, and design of engineering systems," Journal of Mechanical Design, vol. 135, no. 6, pp. 189197, 2013.

[29] J. Ma, W. Liu, and P. Miller, "An evidential improvement for gender profiling," Advances in Intelligent and Soft Computing, vol. 164, pp. 29-36, 2012.

[30] J. Ma, W. Liu, P. Miller, and H. Zhou, "An evidential fusion approach for gender profiling," Information Sciences, vol. 333, pp. 10-20, 2016.

[31] N. Rikhtegar, N. Mansouri, A. A. Oroumieh, A. YazdaniChamzini, E. K. Zavadskas, and S. Kildienè, "Environmental impact assessment based on group decision-making methods in mining projects," Economic Research, vol. 27, no. 1, pp. 378-392, 2014.

[32] Y. Wang, Y. Dai, Y.-W. Chen, and F. Meng, “The evidential reasoning approach to medical diagnosis using intuitionistic fuzzy Dempster-Shafer theory," International Journal of Computational Intelligence Systems, vol. 8, no. 1, pp. 75-94, 2015.
[33] C. Fu and K.-S. Chin, "Robust evidential reasoning approach with unknown attribute weights," Knowledge-Based Systems, vol. 59, pp. 9-20, 2014.

[34] H.-C. Liu, L. Liu, and Q.-L. Lin, "Fuzzy failure mode and effects analysis using fuzzy evidential reasoning and belief rule-based methodology," IEEE Transactions on Reliability, vol. 62, no. 1, pp. 23-36, 2013.

[35] X. Su, S. Mahadevan, P. Xu, and Y. Deng, "Dependence assessment in human reliability analysis using evidence theory and AHP," Risk Analysis, vol. 35, no. 7, pp. 1296-1316, 2015.

[36] L. A. Zadeh, "A simple view of the Dempster-Shafer theory of evidence and its implication for the rule of combination," $A I$ Magazine, vol. 7, no. 2, pp. 85-90, 1986.

[37] C. K. Murphy, "Combining belief functions when evidence conflicts," Decision Support Systems, vol. 29, no. 1, pp. 1-9, 2000.

[38] J. Ma, W. Liu, D. Dubois, and H. Prade, "Revision rules in the theory of evidence," in Proceedings of the 22nd International Conference on Tools with Artificial Intelligence (ICTAI '10), vol. 1, pp. 295-302, October 2010.

[39] P. Smets, "Analyzing the combination of conflicting belief functions," Information Fusion, vol. 8, no. 4, pp. 387-412, 2007.

[40] A.-L. Jousselme, D. Grenier, and É. Bossé, "A new distance between two bodies of evidence," Information Fusion, vol. 2, no. 2, pp. 91-101, 2001.

[41] W. Liu, "Analyzing the degree of conflict among belief functions," Artificial Intelligence, vol. 170, no. 11, pp. 909-924, 2006.

[42] Y. Deng, D. Wang, Q. Li, and Y.-J. Zhang, "A new method to analyze evidence conflict," Control Theory \& Applications, vol. 28, no. 6, pp. 839-844, 2011.

[43] A.-L. Jousselme and P. Maupin, "Distances in evidence theory: comprehensive survey and generalizations," International Journal of Approximate Reasoning, vol. 53, no. 2, pp. 118-145, 2012.

[44] D. Yong, S. WenKang, Z. ZhenFu, and L. Qi, "Combining belief functions based on distance of evidence," Decision Support Systems, vol. 38, no. 3, pp. 489-493, 2004.

[45] M. Wu and Z. Bai, "Relative entropy and its application," Journal of East China Normal University, vol. 2, pp. 28-35, 1998.

[46] W. Jiang and J. Peng, "New representation method of evidential conflict," Systems Engineering and Electronics, vol. 32, pp. 562$565,2010$.

[47] C. Wen, Y. Wang, and X. Xu, "Fuzzy information fusion algorithm of fault diagnosis based on similarity measure of evidence," Lecture Notes in Computer Science, vol. 5264, no. 2, pp. 506-515, 2008.

[48] D. Cheng, R.-X. Hao, and Y.-Q. Feng, "Embedding even cycles on folded hypercubes with conditional faulty edges," Information Processing Letters, vol. 115, no. 12, pp. 945-949, 2015.

[49] Y.-F. Song, X.-D. Wang, L. Lei, and A.-J. Xue, "Measurement of evidence conflict based on correlation coefficient," Journal on Communications, vol. 35, no. 5, pp. 95-100, 2014.

[50] J. Zhang and R. R. Korfhage, "A distance and angle similarity measure method," Journal of the American Society for Information Science, vol. 50, no. 9, pp. 772-778, 1999. 


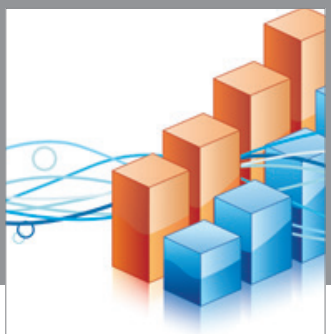

Advances in

Operations Research

vatem alat4

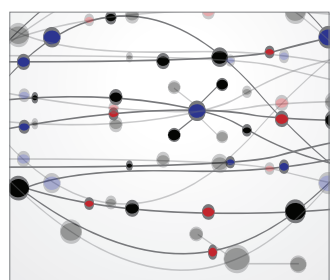

\section{The Scientific} World Journal
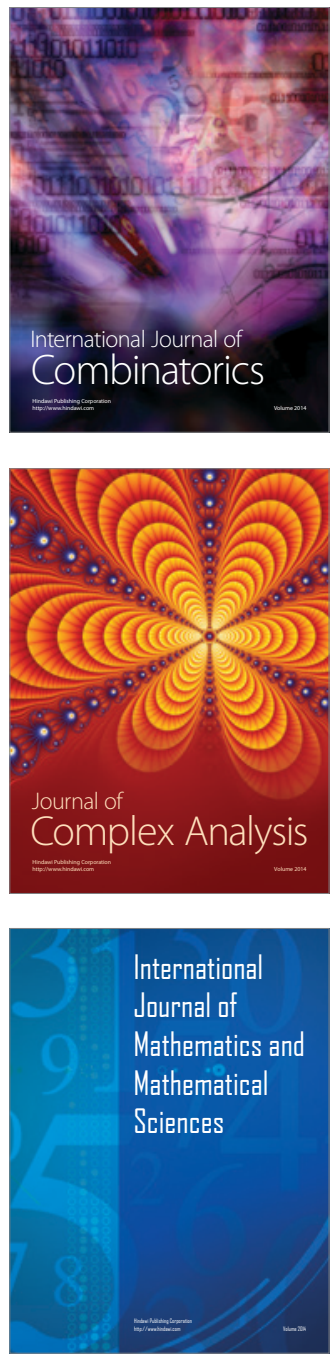
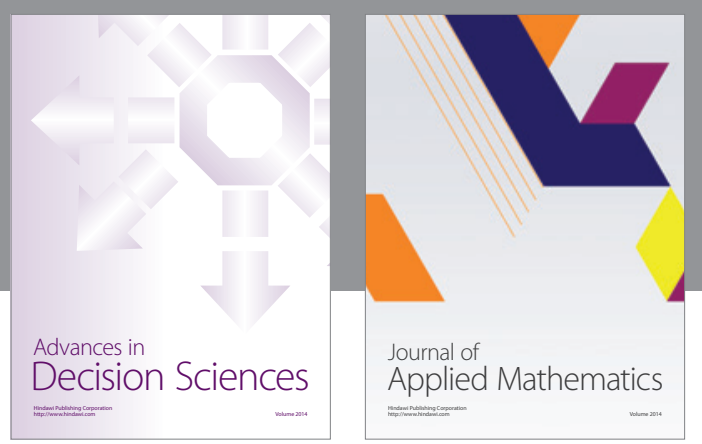

Algebra

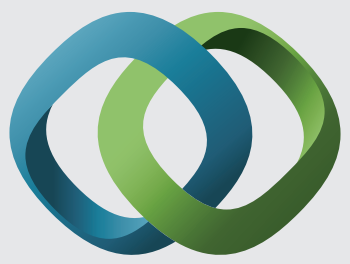

\section{Hindawi}

Submit your manuscripts at

http://www.hindawi.com
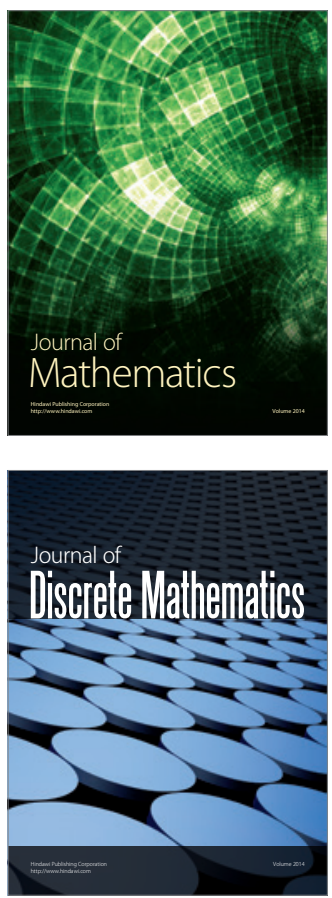

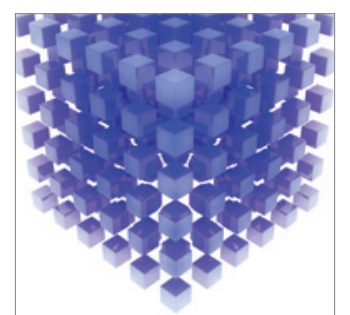

Mathematical Problems in Engineering
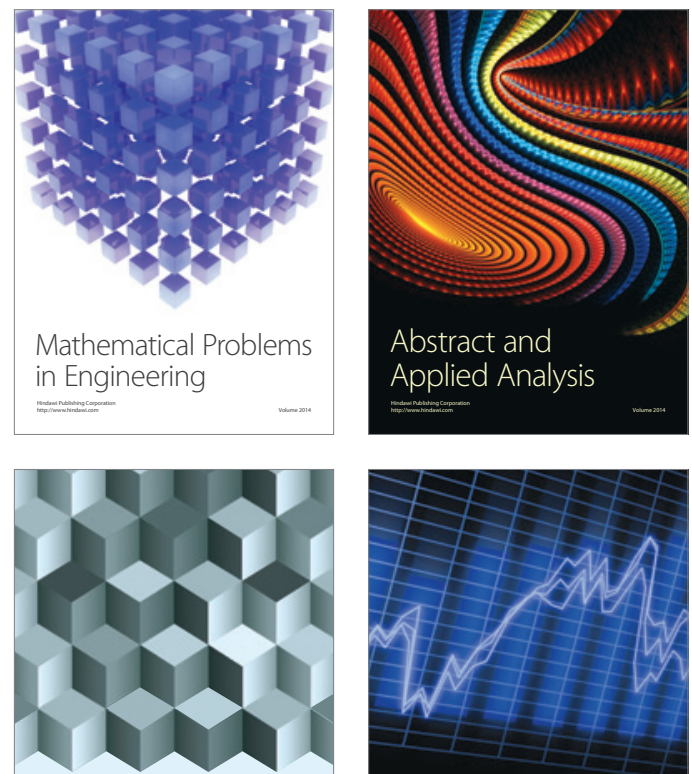

Journal of

Function Spaces

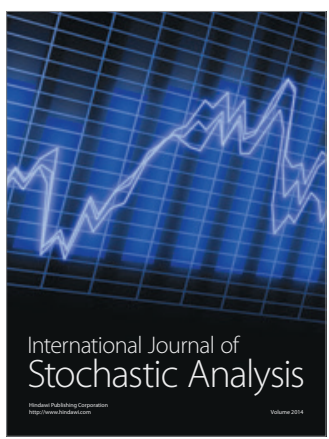

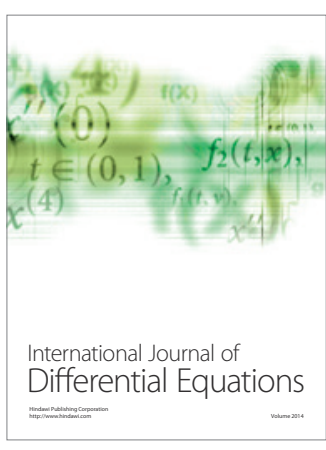
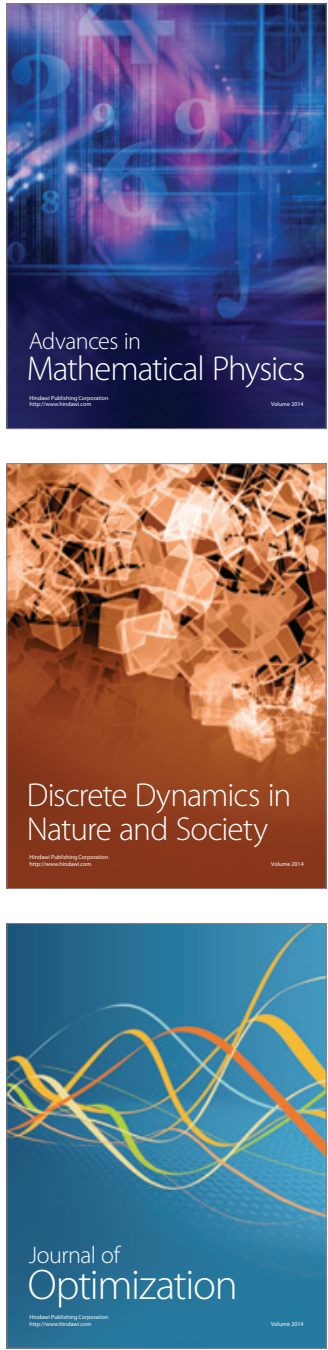